\title{
High mobility group box protein 1 neutralization therapy in ovine bacteremia: Lessons learned from an ovine septic shock model incorporating intensive care support
}

\author{
NATALIE E. STEVENS ${ }^{1,2}$, CORALIE H. NASH ${ }^{3}$, CARA K. FRASER $^{4}$, \\ TIM R. KUCHEL ${ }^{4}$, MATTHEW J. MAIDEN ${ }^{3,5,6}$, MARIANNE J. CHAPMAN ${ }^{3,6}$, \\ KERRILYN R. DIENER ${ }^{1,7,8}$ and JOHN D. HAYBALL ${ }^{1,7,8}$
}

\footnotetext{
${ }^{1}$ Experimental Therapeutics Laboratory, University of South Australia Cancer Research Institute, Adelaide, SA 5000;

${ }^{2}$ Future Industries Institute, University of South Australia, Mawson Lakes, SA 5095;

${ }^{3}$ Discipline of Acute Care Medicine, University of Adelaide, Adelaide, SA 5000; ${ }^{4}$ Preclinical, Imaging and

Research Laboratories, South Australian Health and Medical Research Institute, Gilles Plain, SA 5086;

${ }^{5}$ Intensive Care Unit, Barwon Health, Geelong, VIC 3220; ${ }^{6}$ Intensive Care Unit, Royal Adelaide Hospital, Adelaide, SA 5000; ${ }^{7}$ School of Pharmacy and Medical Sciences, University of South Australia, Adelaide, SA 5001;

${ }^{8}$ Robinson Research Institute and Adelaide Medical School, University of Adelaide, Adelaide, SA 5000, Australia
}

Received October 12, 2018; Accepted February 19, 2019

DOI: $10.3892 /$ etm.2019.7961

\begin{abstract}
Sepsis is a highly complex and often fatal syndrome which varies widely in its clinical manifestations, and therapies that target the underlying uncontrolled immune status in sepsis are needed. The failure of preclinical approaches to provide significant sepsis survival benefit in the clinic is often attributed to inappropriate animal disease models. It has been demonstrated that high mobility group box protein 1 (HMGB1) blockade can reduce inflammation, mortality and morbidity in experimental sepsis without promoting immunosuppression. Within this study, we explored the use of ovine anti-HMGB1 antibodies in a model of ovine septic shock incorporating intensive care supports (OSSICS). Results: Septic sheep exhibited elevated levels of HMGB1 within $12 \mathrm{~h}$ after the induction of sepsis. In this study, sepsis was induced in six anaesthetized adult Border Leicester x Merino ewes via intravenous instillation of E. coli
\end{abstract}

Correspondence to: Dr Natalie E. Stevens, Future Industries Institute, University of South Australia, Building X, Mawson Lakes Campus, Mawson Lakes, SA 5095, Australia

E-mail: natalie.stevens@unisa.edu.au

Abbreviations: CO, cardiac output; CVP, central venous pressure; DAMP, damage-associated molecular pattern; HMGB1, high mobility group box 1; HR, heart rate; MAP, mean arterial pressure; NorA, noradrenaline (Norepinephrine); OSSICS, ovine septic shock incorporating intensive care support; pAb(s), polyclonal antibody(-ies); PCV, packed cell volume; RCT, randomized controlled trial; SOFA, sequential organ failure assessment; SVRI, systemic vascular resistance index

Key words: animal models, sepsis, sheep, pAb(s), HMGB1 and sheep monitored according to intensive care unit standard protocols for $26 \mathrm{~h}$, with the requirement for noradrenaline as the primary endpoint. Septic sheep exhibited a hyperdynamic circulation, renal dysfunction, deranged coagulation profile and severe metabolic acidosis. Sheep were assigned a severity of illness score, which increased over time. While a therapeutic effect of intravenous anti-HMGB1 antibody could not be observed in this model due to limited animal numbers, a reduced bacterial dose induced a septic syndrome of much lower severity. With modifications including a reduced bacterial dose, a longer timeframe and broad spectrum antibiotics, the OSSICS model may become a robust tool for preclinical assessment of sepsis therapeutics.

\section{Introduction}

Sepsis is broadly defined as a dysregulated immune response to a pathogenic insult which damages host tissues, and represents a highly complex and often fatal syndrome which varies widely in its clinical presentation. Currently, sepsis treatment is primarily limited to supportive medications and broad spectrum antibiotics, however currently no clinically validated approach exists to treat the underlying immune dysregulation in sepsis. The majority of immune-modulating therapeutics that show efficacy in preclinical sepsis models do not exhibit similar clinical benefits (1) which is often attributed to the pervasive use of inappropriate animal models. The use of relevant animal models is imperative to gain insights into sepsis pathophysiology, to improve treatment paradigms and to investigate long-term effects in survivors. Small animal models favor quick, cost effective and easily executed studies but larger animal models are often more appropriate to measure multiple clinical parameters within more relevant physiological contexts to humans. 
The availability of genetically defined rodent strains has enabled in-depth evaluation of immunological parameters that are not achievable in large animal species (2). Despite controversy surrounding the applicability of murine studies to human diseases (3), recent evidence suggests that mouse models parallel many aspects of human inflammatory and immunological diseases (4). Nevertheless, large animal models including pigs, sheep and dogs are more likely to recapitulate pathophysiologic changes seen in humans (5). The reclassification of sepsis according to Sepsis-3 has ignited a renewed focus on organ damage rather than systemic inflammatory criteria as the most useful diagnostic and prognostic measures for sepsis (6). Consequently, preclinical approaches are adopting a similar shift away from immunological endpoints towards more integrated measures $(7,8)$. Ovine models are particularly advantageous for investigation of physiological outcomes due to the hemodynamic similarity between sheep and humans (5), the availability of sheep, and the ability to repurpose clinical instrumentation. Sheep are comparable to humans for most common coagulation tests (9), and exhibit similar metabolic changes in response to sepsis (10). Instrumented septic ovine models have been used to study acute lung injury (11), fluid resuscitation (12) and renal inflammation (13). Recently, an ovine septic shock incorporating intensive care supports (OSSICS) model which recapitulates multiple pathophysiological hallmarks of human septic shock (14) has been used to investigate renal pathology (15) and thyroid hormone therapy (16) in sepsis. However, the utility of this model for studying the physiologic effects of immunomodulatory therapies has not been established.

High mobility group box protein 1 (HMGB1) is a potential therapeutic target in sepsis with a wide therapeutic window (17). Normally located within the nucleus, HMGB1 is released during sepsis and promotes inflammation as a damage associated molecular pattern (DAMP). Preclinical modelling has shown that methods used to neutralize or inhibit HMGB1 activity reduce mortality (18).

Previously, our laboratory has demonstrated that ovine anti-HMGB1 polyclonal antibodies (pAbs) can reduce mortality in murine endotoxemia (19) and polymicrobial sepsis (20) in a similar manner to other studies (21). While the effects of anti-HMGB1 pAb therapy on the immune phenotype of sepsis may be assessed in the murine model, little can be ascertained about the impact of such therapy on sepsis pathophysiology. Therefore, we aimed to determine the suitability of the OSSICS model for the evaluation of anti-HMGB1 pAb using clinically-relevant parameters.

\section{Materials and methods}

Ovine polyclonal anti-HMGBI production. Ovine anti-HMGB1 pAbs were generated via immunization of sheep with recombinant HMGB1 in Freund's adjuvant and purified from anti-HMGB1 serum via Protein G chromatography as previously described (19). Neutralization capacity of ovine anti-HMGB1 pAbs were evaluated as previously described (19). Purified pAbs were sterile filtered and adjusted to $5 \mathrm{mg} / \mathrm{ml}$ in PBS for administration.

HMGB1 and anti-HMGB1 quantitation by ELISA. Sheep serum samples were analyzed by a human HMGB-1 ELISA kit (Cloud-Clone Corp; standard curve range 4,000-63 pg/ml) according to the manufacturer's instructions. Anti-HMGB1 was quantified in sheep serum using a custom ELISA method. Briefly, ELISA plates were coated with recombinant HMGB1 antigen $(5 \mu \mathrm{g} / \mathrm{ml})$ produced as previously described (19) and blocked with BSA. Sheep serum samples were diluted 1:2 and bound ovine antibodies were detected with anti-ovine IgG-HRP (Donkey anti-sheep IgG A3415; Sigma Aldrich) and OPD substrate (SigmaFAST; Sigma Aldrich). Readings were compared to a standard curve prepared from an aliquot of anti-HMGB1 pAb preparation.

Sheep preparation and monitoring. All sheep studies were approved by at The South Australian Health and Medical Research Institute (Adelaide, Australia) Animal Ethics Committee and conducted following institutional and national ethical guidelines according to the 'Australian code for the care and use of animals for scientific purposes (2013)'. Border Leicester x Merino ewes (2.4-4 years old) were group housed at The South Australian Health and Medical Research Institute and given free access to food and water. A model of OSSICS was performed on six sheep previously published protocols $(15,16)$. Briefly, anesthesia was induced in overnight fasted randomly selected sheep by intravenous (IV) injection of Thiopentone $(15 \mathrm{mg} / \mathrm{kg})$ before intubation and ventilation with isoflurane (2\%) in oxygen the morning following fasting (8-10 am). Catheters were placed under surgical conditions: an arterial catheter (Angiocath $1.7 \times 133 \mathrm{~mm}$; Becton Dickinson) was placed into the right carotid artery, and a quad-lumen central venous catheter (8.5 Frx20 cm; Arrow International Inc.) and a large-bore single lumen catheter $(8.5 \mathrm{Frx} 10 \mathrm{~cm}$; Arrow International Inc.) were both inserted into the right jugular vein. The large-bore single lumen catheter was used for the placement of a pulmonary artery catheter (Swan Ganz CCOmbo 7.5 Frx $110 \mathrm{~cm}$; Edwards Lifesciences) performed under radiology imaging.

A tracheostomy tube was inserted into the proximal trachea (Portex, ID 9.0, $24 \mathrm{~cm}$; Smith's Medical) and a rumen tube (16 gauge, Bard Medical) was inserted via the nose. An indwelling urethral catheter (14Fr, Covington; Bard Medical) was placed into the bladder and attached to a burette for measurement. Sheep were moved onto a surgical table, lying prone with head and neck supported in an upright position to prevent edemous fluid accumulation in the lower limbs, and mechanically ventilated (Puritan Bennett 7200). Central venous and arterial catheters were attached to a pressure monitor (Intellivue MP 50; Phillips) and a pulmonary artery catheter to cardiac output monitor (Vigilance Monitor, Edwards Lifesciences). Cardiac output was continuously monitored and indexed to sheep body surface area according to the calculation (weight $(\mathrm{kg}) 0.67 \mathrm{x} 0.0842$ ).

All sheep were monitored by a dedicated intensive care research nurse throughout the study (Table I), and mean arterial pressure (MAP) was maintained according to the protocol described in Table II. Previous studies have demonstrated that anti-HMGB1 pAbs are efficacious in reducing mortality in murine endotoxemia within $24 \mathrm{~h}$ (19), thus a short time frame was adopted in the current study to allow comparison to previous OSSICS cohorts. Sheep were euthanized under 
Table I. Sheep monitoring and maintenance.

\begin{tabular}{|c|c|c|}
\hline Parameter & Monitoring & Maintenance \\
\hline Sedation & $\begin{array}{l}\text { Depth of sedation continuously monitored; medication } \\
\text { dose recorded hourly. }\end{array}$ & $\begin{array}{l}\text { IV infusion of midazolam }(0.1-0.5 \mathrm{mg} / \mathrm{kg} / \mathrm{h}) \\
\text { and ketamine }(1-5 \mathrm{mg} / \mathrm{kg} / \mathrm{h})\end{array}$ \\
\hline Ventilation & $\begin{array}{l}\text { Respiratory rate, tidal volume, peak and plateau } \\
\text { pressures and } \mathrm{O}_{2} \text { and } \mathrm{CO}_{2} \text { saturation recorded hourly. } \\
\text { Tracheal suctioning was performed hourly or } \\
\text { as required. }\end{array}$ & $\begin{array}{l}\text { Synchronised intermittent mandatory ventilation with } \\
10 \mathrm{ml} / \mathrm{kg} \text { tidal volume and positive end expiratory } \\
\text { pressure of } 5 \mathrm{~cm} \mathrm{H}_{2} \mathrm{O} \text {. } \\
\text { Respiration adjusted to maintain end tidal } \mathrm{CO}_{2} \text { of } \\
30 \mathrm{mmHg} \text {. Fraction of inspired oxygen was adjusted } \\
\text { to maintain pulse } \mathrm{O}_{2} \text { saturation }>95 \% \text {. }\end{array}$ \\
\hline Hemodynamics & $\begin{array}{l}\mathrm{HR}, \mathrm{CVP} \text { and pulmonary arterial pressure were } \\
\text { recorded hourly. } \\
\text { MAP and CO recorded every } 10 \mathrm{~min} .\end{array}$ & $\begin{array}{l}\text { Parenteral fluid was administered at } 3 \mathrm{ml} / \mathrm{kg} / \mathrm{h} \text { via } \\
\text { peristaltic pump. } \\
\text { CVP was maintained } \geq 5 \mathrm{mmHg} \text { via fluid bolus } \\
\text { administration ( } 250 \mathrm{ml} \text { saline). } \\
\text { MAP was maintained } \geq 75 \mathrm{~mm} / \mathrm{Hg} \text { (Table II). }\end{array}$ \\
\hline Temperature & Recorded every hour. & $\begin{array}{l}\text { Temperature was maintained }<42^{\circ} \mathrm{C} \text { with the } \\
\text { application of cool damp drapes to the back }\end{array}$ \\
\hline Blood analysis & - & $\begin{array}{l}\text { Samples taken every } 2 \mathrm{~h} \text { for cytokine and lactate } \\
\text { analysis, every } 4 \mathrm{~h} \text { for blood gas analysis and every } \\
6 \mathrm{~h} \text { for pathology analysis including coagulation, } \\
\text { hematological and biochemical tests. }\end{array}$ \\
\hline Fluid balance & Fluids administered and urine output recorded hourly. & $\begin{array}{l}\text { Total urine collected at } 0,12 \text { and } 26 \mathrm{~h} \text { was subjected } \\
\text { to protein and creatinine analysis. }\end{array}$ \\
\hline
\end{tabular}

$\mathrm{HR}$, heart rate; CVP, central venous pressure; MAP, mean arterial pressure; $\mathrm{CO}$, cardiac output.

Table II. Ovine model hemodynamic management protocol.

\begin{tabular}{ll}
\hline Blood pressure parameters & \multicolumn{1}{c}{ Action } \\
\hline MAP $<75 \mathrm{mmHg}$ and & $250 \mathrm{ml}$ saline bolus \\
CVP $<5 \mathrm{mmHg}$ & \\
$75 \mathrm{mmHg}$ and CVP $\geq 5 \mathrm{mmHg}$ & NorA infusion as below; \\
& adjust every $2 \mathrm{~min}$ \\
MAP <55 mmHg & $\uparrow 10 \mu \mathrm{g} / \mathrm{min}$ NorA \\
MAP 55-59 mmHg & $\uparrow 5 \mu \mathrm{g} / \mathrm{min}$ NorA \\
MAP 60-64 mmHg & $\uparrow 3 \mu \mathrm{g} / \mathrm{min}$ NorA \\
MAP 65-69 mmHg & $\uparrow 2 \mu \mathrm{g} / \mathrm{min}$ NorA \\
MAP 70-74 mmHg & $\uparrow 1 \mu \mathrm{g} / \mathrm{min}$ NorA \\
MAP 75-80 mmHg & No change to NorA dose \\
MAP 81-85 mmHg & $\downarrow 1 \mu \mathrm{g} / \mathrm{min}$ NorA \\
MAP 86-90 mmHg & $\downarrow 2 \mu \mathrm{g} / \mathrm{min}$ NorA \\
MAP 91-95 mmHg & $\downarrow 3 \mu \mathrm{g} / \mathrm{min}$ NorA \\
MAP 96-00 mmHg & $\downarrow 5 \mu \mathrm{g} / \mathrm{min}$ NorA \\
MAP >100 mmHg & $\downarrow 10 \mu \mathrm{g} / \mathrm{min}$ NorA \\
\hline
\end{tabular}

MAP, mean arterial pressure; NorA, noradrenaline.

anesthesia at $26 \mathrm{~h}$ post- $E$. coli administration by IV injection of pentobarbitone $(6.5 \mathrm{~g})$. Death was apparent by unrecordable arterial blood pressure, and confirmed by a trained intensive care nurse. All laboratory and biochemical measurements were conducted by technicians blinded to the treatment group identity.

Preparation and administration of E. coli and anti-HMGB1 polyclonal antibodies. E. coli (Serotype O6, Biotype 1; ATCC 25922) inoculum was prepared from colonies (8-10) grown overnight on fresh Columbia horse blood agar (bioMérieux Australia) emulsified in $100 \mathrm{ml}$ sterile saline to an optical density of 0.25 at $550 \mathrm{~nm}$ (empirically determined to yield $1.5 \times 10^{8} \mathrm{CFU} / \mathrm{ml}$ ). The number of $\mathrm{CFUs} / \mathrm{ml}$ of each inoculum was determined by serial dilution and plating onto Columbia Horse Blood agar.

The inoculum $(1 \mathrm{ml} / \mathrm{kg})$ was administered to prepared, anaesthetized sheep as an IV infusion in the over one hour to achieve $1-1.5 \times 10^{8} \mathrm{CFU} / \mathrm{kg}$ based on previous studies $(15,16)$. Anti-HMGB1 antibodies $(10 \mathrm{mg} / \mathrm{kg})$ were administered via IV infusion over one hour commencing two hours after the induction of sepsis. This dose rate was based on previous murine studies and is in molar excess (180-fold) of detectable plasma HMGB1. Placebo-treated sheep were administered IV saline in place of the antibody infusion. Bacterial doses and treatments administered to the six experimental sheep are summarized in Table III.

Statistical analysis. Analysis was performed using GraphPad Prism V3.0 software. Measurements at individual time points were compared using student's two-tailed t-tests and time course analysis was performed by two-way analysis of variance. 
Table III. Characteristics of sheep and survival outcomes within the study as compared to archive data.

\begin{tabular}{|c|c|c|c|c|c|c|}
\hline Sheep & $\begin{array}{l}\text { Weight, } \\
\text { kg }\end{array}$ & $\begin{array}{l}\text { E. coli dose, } \\
\text { CFU/kg }\end{array}$ & Treatment & $\begin{array}{l}\text { Survival } \\
\text { time, } \mathrm{h}\end{array}$ & $\begin{array}{c}\text { Cumulative } \\
\text { NorA administered, } \\
\mu \mathrm{g} / \mathrm{kg}\end{array}$ & $\begin{array}{l}\text { NorA dose } \\
\text { rate mean } \\
\text { AUC, } \mu \mathrm{g} / \mathrm{kg}\end{array}$ \\
\hline 1 & 68 & $1.08 \times 10^{8}$ & Placebo (normal saline) & 26 & 26.6 & $13.3^{\mathrm{a}}$ \\
\hline 2 & 74 & $0.94 \times 10^{8}$ & Placebo (normal saline) & 26 & 0 & \\
\hline 3 & 69 & $1.47 \times 10^{8}$ & Placebo (normal saline) & 26 & 718.5 & 715 \\
\hline 4 & 58 & $1.62 \times 10^{8}$ & $10 \mathrm{mg} / \mathrm{kg}$ anti-HMGB $1 \mathrm{pAbs}$ & 26 & 556.2 & $794^{\mathrm{b}}$ \\
\hline 5 & 71 & $1.50 \times 10^{8}$ & $10 \mathrm{mg} / \mathrm{kg}$ anti-HMGB $1 \mathrm{pAbs}$ & 12 & 234.4 & \\
\hline 6 & 76 & $1.58 \times 10^{8}$ & $10 \mathrm{mg} / \mathrm{kg}$ anti-HMGB $1 \mathrm{pAbs}$ & 25 & 869.4 & \\
\hline $\begin{array}{l}\text { Archive data } \\
(\mathrm{n}=8 ; \text { mean } \pm \mathrm{SD})\end{array}$ & $63 \pm 8$ & $1.08 \pm 0.5 \times 10^{8}$ & Placebo (normal saline) & $25 \pm 2.1$ & $330 \pm 403$ & 340 \\
\hline
\end{tabular}

${ }^{\text {a} A p p l i e s ~ t o ~ s h e e p ~} 1$ and 2; bapplies to sheep 4-6. SD, standard deviation; NorA, noradrenaline; AUC, area under the curve; HMGB1, high mobility group box 1 .

\section{Results}

Ovine septic shock induces elevation of plasma HMGB1. First we determined whether HMGB1 release kinetics were similar between sheep and humans. Banked plasma samples from previous OSSICS subjects (see references 15 and 16) and analyzed for HMGB1 content by commercial ELISA. Elevated HMGB1 was detectable (Fig. 1) from $12 \mathrm{~h}$ post E. coli infusion $\left(1.08 \pm 0.05 \times 10^{8} \mathrm{CFU} / \mathrm{kg}\right)$ while no HMGB1 elevation was observed in non-septic animals. Significantly, plasma HMGB1 levels at $26 \mathrm{~h}$ were three-fold higher in septic sheep than non-septic sheep, or those measured at the onset of sepsis (Fig. 1). Septic sheep exhibited HMGB1 levels between 2-6 $\mathrm{ng} / \mathrm{ml}$ over the course of sepsis. These results support the use of the ovine septic shock model for the study of anti-HMGB1 therapy in sepsis.

Anti-HMGB1 therapy did not reduce the development of shock or noradrenaline requirement in the OSSICS model. The requirement for vasopressor therapy in the form of noradrenaline (NorA) to maintain adequate blood pressure has previously been used as the primary outcome measure in the OSSICS model. Within this study, shock was generated in six sheep and compared to data from previous studies utilising the same protocol. Characteristics of study sheep and outcomes are depicted in Table III. Initially, two sheep (\#1 and \#2) were administered the same $E$. coli dose as used in previous studies (1x $\left.10^{8} \mathrm{CFU} / \mathrm{kg}\right)(15,16)$, however the sheep required little NorA and did not progress to prolonged septic shock. To replicate a degree of sickness severity observed in previous studies, an increased $E$. coli dose $\left(1.5 \times 10^{8} \mathrm{CFU} / \mathrm{kg}\right)$ was employed for the remaining sheep within the study (\#3-6). Three sheep were administered anti-HMGB1 pAbs (10 mg/kg; sheep 4-6) two hours after the induction of sepsis. Two early deaths occurred within this group, as reflected by the recorded survival time. Hemodynamic parameters were evaluated in sheep for $1 \mathrm{~h}$ before the induction of sepsis and for $26 \mathrm{~h}$ thereafter (Fig. 2). Placebo-treated sheep from previous studies $(n=8$; hereafter referred to as archive data) displayed a rapid decrease in MAP and SVRI within $2 \mathrm{~h}$ of bacterial infusion before increasing to

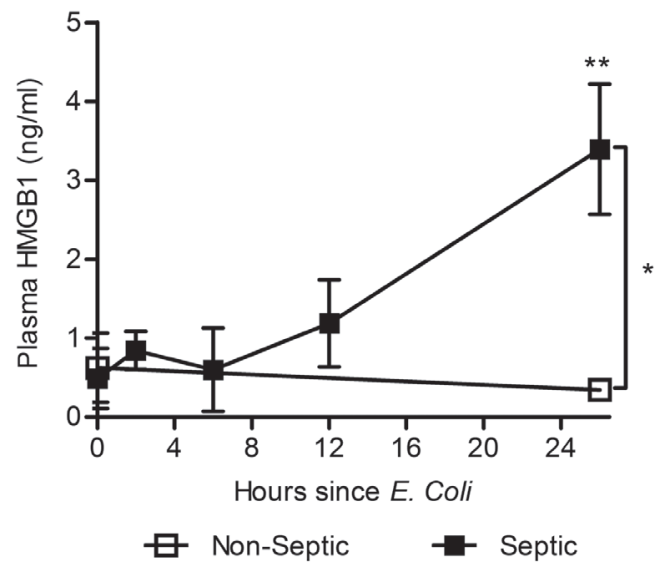

Figure 1. Significant elevation of plasma HMGB1 is detectable at $26 \mathrm{~h}$ in septic sheep. Kinetic plasma samples from sheep administered $1 \times 10^{8} \mathrm{CFU} / \mathrm{kg}$ E. coli $(\mathrm{n}=4)$ and non-septic control animals $(\mathrm{n}=4)$ in previous studies were assessed for HMGB1 content via ELISA. The mean \pm standard error of the mean of individual time points are represented. ${ }^{*} \mathrm{P}<0.05,{ }^{* *} \mathrm{P}<0.01$. HMGB1, high mobility group box 1 .

a stable level within $4 \mathrm{~h}$ of infusion (Fig. 2A and B). Between 6-8 $\mathrm{h}$ after infusion, MAP and SVRI gradually declined until a MAP $<75 \mathrm{mmHg}$ was achieved and NorA therapy was initiated (Fig. 2C). Two sheep within the current study were administered $1 \times 10^{8} \mathrm{CFU} / \mathrm{kg}$ E. coli and these sheep displayed a markedly reduced need for NorA compared to archive data. One sheep with an increased bacterial dose $\left(1.5 \times 10^{8} \mathrm{CFU} / \mathrm{kg}\right)$ exhibited consistently low MAP and SVRI and had high NorA requirements. Infusion of anti-HMGB1 pAbs two hours post-challenge in three sheep that received the increased bacterial dose of $1.5 \times 10^{8} \mathrm{CFU} / \mathrm{kg}$ did not appear to impact on NorA requirements compared to the equivalently-dosed control sheep. Urine output (Fig. 2D) in the OSSICS model appeared consistent with archive data, where measured urine output was between 1-4 ml/hour/kg and subsequently fell to less than $1 \mathrm{ml} / \mathrm{h} / \mathrm{kg}$ by $8 \mathrm{~h}$ post-challenge.

Administration of anti-HMGB1 pAb did not alter detectable HMGB1 levels in septic sheep plasma. In this preclinical 
A

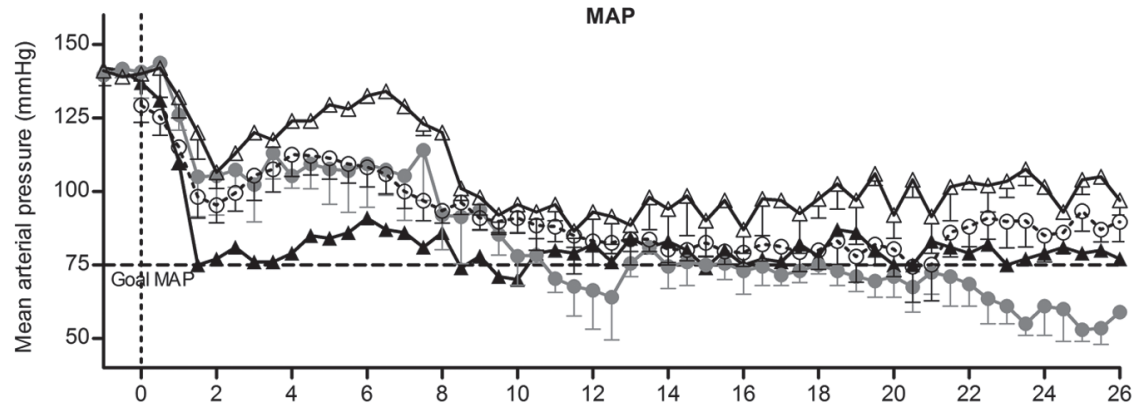

B

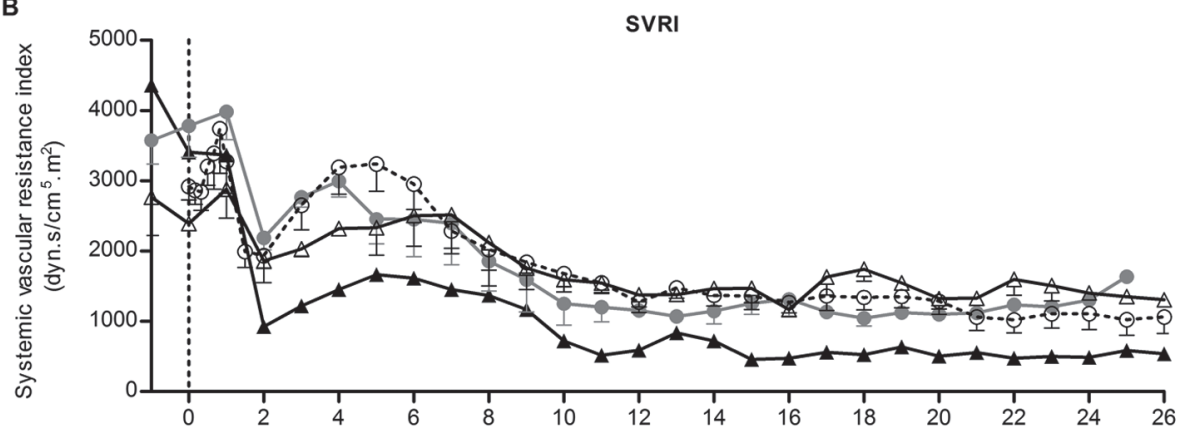

C

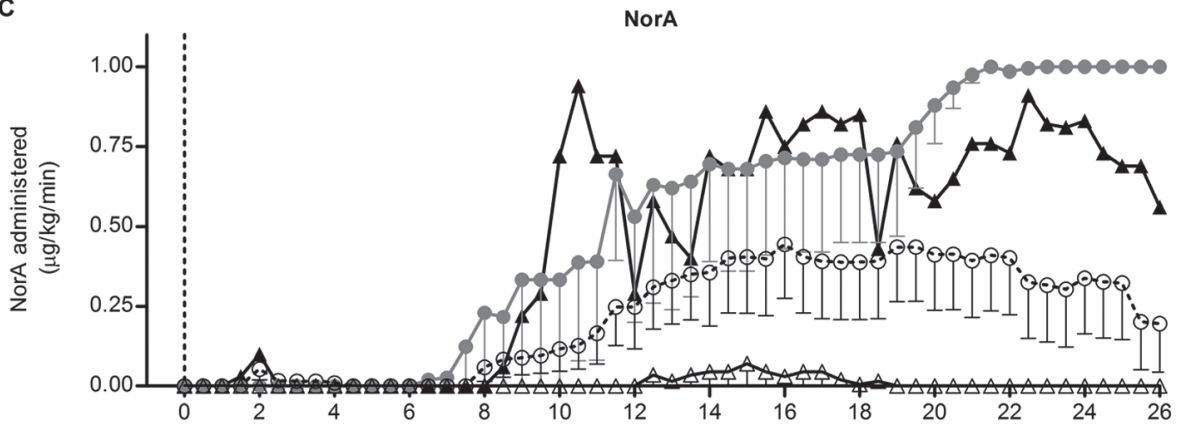

D

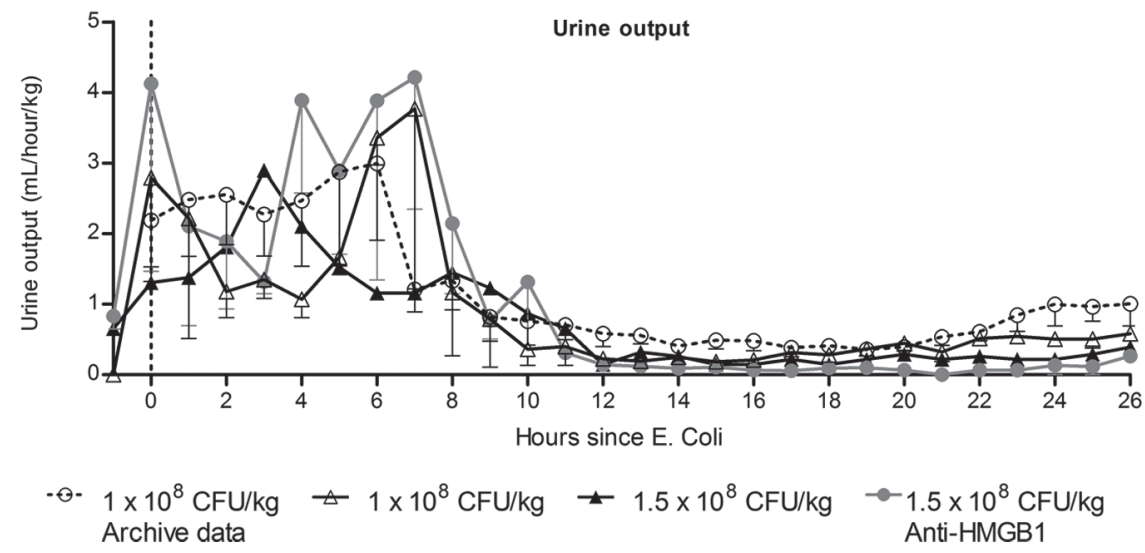

Figure 2. Measured hemodynamic parameters in an intensive care support ovine septic shock model. Sheep were anesthetized and catheterized to enable measurement of vascular pressure and urine output. After $1 \mathrm{~h}$, sepsis was induced (indicated by dotted vertical line) by the IV infusion of $1 \times 10^{8} \mathrm{CFU} / \mathrm{kg} E$.coli $(\mathrm{n}=2)$, or $1.5 \times 10^{8} \mathrm{CFU} / \mathrm{kg}$ E. coli with placebo (saline) treatment (n=1) or $10 \mathrm{mg} / \mathrm{kg}$ anti-HMGB1 pAb (n=3). (A) MAP at 10 min intervals and (B) hourly SVRI to body surface area are represented. (C) NorA requirements were recorded at $10 \mathrm{~min}$ intervals and (D) urine output were recorded hourly. Data are presented as the mean-standard error of the mean of measurements for each group with archive data from placebo-treated (saline) sheep (n=8) included for comparison HMGB1, high mobility group box 1; MAP, mean arterial pressure; NorA, noradrenaline; SVRI, systemic vascular resistance index.

model, a dose rate of $10 \mathrm{mg} / \mathrm{kg}$ anti-HMGB1 $\mathrm{pAb}$ was selected based on previous murine studies and on concentrations of HMGB1 detected within the circulation of septic sheep. In order to establish whether sufficient anti-HMGB1 pAb was administered in this study to observe effects, kinetic serum samples from the three Ab dosed sheep were analyzed by
ELISA to detect anti-HMGB1 ovine IgG (Fig. 3A) and circulating HMGB1 (Fig. 3B). Anti-HMGB1 pAbs were detectable two hours following $\mathrm{Ab}$ infusion at $18 \mu \mathrm{g} / \mathrm{ml}(18 \mathrm{mg} / \mathrm{l})$, and decreased thereafter. As one sheep died $11 \mathrm{~h}$ following E. coli infusion, measurements at later time points are the mean values of two remaining sheep. These sheep exhibited vastly 


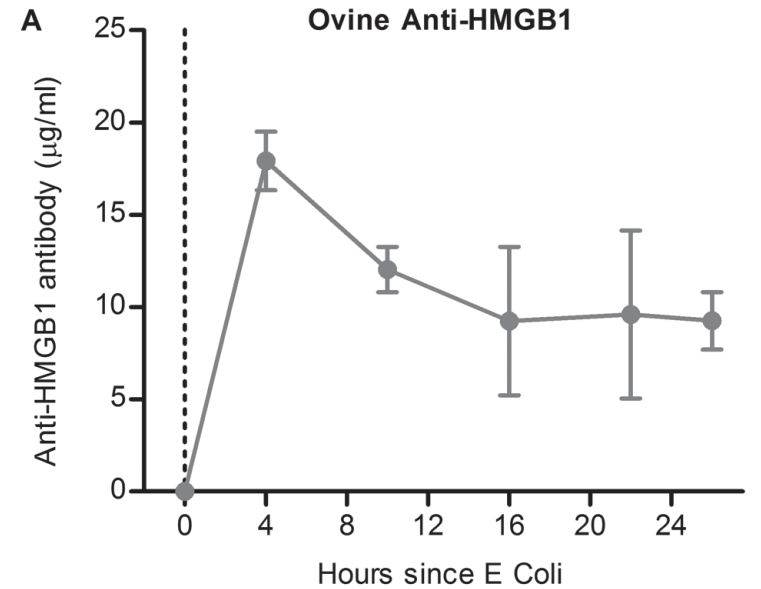
- $\quad 1 \times 10^{8} \mathrm{CFU} / \mathrm{kg}$ Archive data; $n=4$

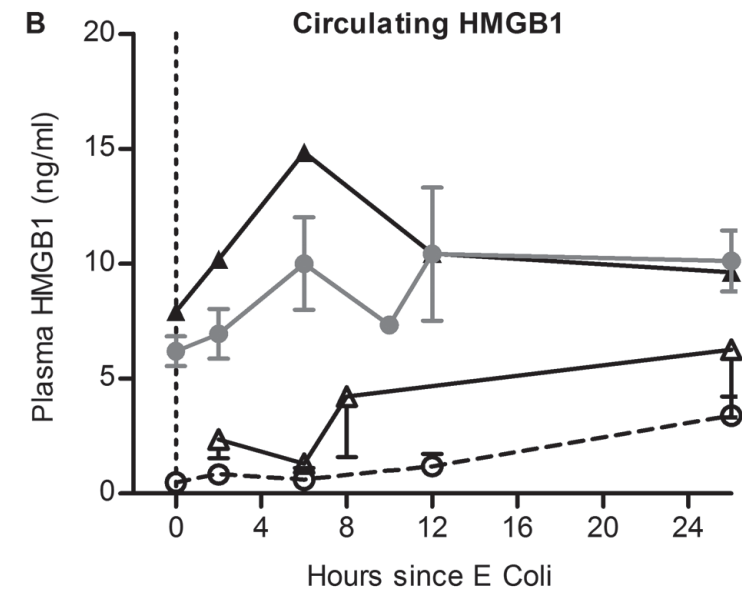

$\rightarrow \quad 1.5 \times 10^{8} \mathrm{CFU} / \mathrm{kg}$ $n=1$

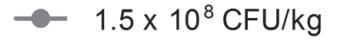

Anti-HMGB $1 ; n=3$

Figure 3. Kinetics of circulating anti-HMGB1 pAb and impact on plasma HMGB1 in the ovine septic shock incorporating intensive care support model. Sheep were anesthetized and sepsis was induced (indicated by dotted vertical line) by the IV infusion of $1 \times 10^{8} \mathrm{CFU} / \mathrm{kg}$ E. coli $(\mathrm{n}=2)$, or $1.5 \times 10^{8} \mathrm{CFU} / \mathrm{kg} E$. coli with placebo treatment $(\mathrm{n}=1)$ or $10 \mathrm{mg} / \mathrm{kg}$ anti-HMGB1 pAb $(\mathrm{n}=3)$ administered $2 \mathrm{~h}$ following bacterial challenge. Levels of circulating $(\mathrm{A})$ ovine anti-HMGB1 pAb and (B) HMGB1 were determined by ELISA analysis. HMGB1, high mobility group box 1; pAb, polyclonal antibody.

different kinetics of pAb clearance, as the detected concentration decreased rapidly in one sheep and remained constant in another, which may be due to metabolic variability in the sheep following sepsis induction.

Sepsis-associated alterations in hematological, coagulative and metabolic parameters were not improved by anti-HMGBI treatment. In the current study, blood and plasma samples drawn from septic sheep were subjected to standard pathology testing and compared to archive values from placebo-treated septic sheep. Ovine septic shock has previously been associated with a progressive decline in packed cell volume (PCV). In the current study, pre-operative samples were drawn which showed increased PCV and hemoglobin (data not shown) compared to pre-septic baseline samples. Measured PCV sharply increased in all groups $2 \mathrm{~h}$ post challenge and declined similarly for all groups for the duration of the study (Fig. 4Aa). Conversely to $\mathrm{PCV}$, measured $\mathrm{WBC}$ counts increased from pre-operative to baseline time points (Fig. 4Ab), potentially due to inflammation caused by surgical catheter placement or isoflurane exposure. WBC counts decreased sharply by $80 \% 2 \mathrm{~h}$ post challenge before gradually increasing over time. Further analysis showed that this decrease was due to marked depletion of neutrophils, which decreased by $98 \%$ from baseline values (Fig. 4Ac), whereas lymphocytes (Fig. 4Ad) and other granulocytes (data not shown) did not change significantly.

Sheep that received the lower E. coli dose $\left(1 \times 10^{8} \mathrm{CFU} / \mathrm{kg}\right)$ exhibited mild coagulopathy, with only a modest increase in PT (Fig. 4Ba) and APTT (Fig. 4Bb) and mild fibrinogen depletion (Fig. 4Bc) from baseline values compared to archive data. These sheep also exhibited reduced platelet depletion (Fig. 4Bd), although this was not consistent throughout the septic period. Sheep treated with anti-HMGB1 pAbs exhibited more profound clotting dysfunction than a placebo-treated sheep given the same $E$. coli dose, however these did not appear altered from archive data and low subject numbers preclude statistical comparison. As anti-HMGB1 pAb treated sheep exhibited the highest mortality of the groups, these results support the notion of coagulation function tests as a marker of disease severity in ovine septic shock, however further investigation is required to determine if anti-HMGB1 therapy may influence clotting dysfunction.

Creatinine, lactate and $\mathrm{pH}$ were monitored throughout the experimental period as a measure of metabolic status. Plasma creatinine (Fig. $4 \mathrm{Ca}$ ) increased in all groups from $2 \mathrm{~h}$ post challenge, however a marked increase was observed in sheep treated with anti-HMGB1 pAbs. Similarly, the anti-HMGB1 pAb group exhibited the most elevated lactate (Fig. $4 \mathrm{Cb}$ ) measurements and the highest degree of acidosis (Fig. $4 \mathrm{Cc}$ ). The group that received a low $E$. coli dose exhibited mild increases in creatinine, and no increase in plasma lactate. Correspondingly, the development of acidosis was largely absent in this group, as $\mathrm{pH}$ only decreased 0.06 units below baseline. These results support the notion that metabolic parameters are useful clinical tests in ovine septic shock that reflect disease severity.

Modified SOFA scores may reflect severity of illness in the OSSICS model. Clinically, the sequential organ failure assessment (SOFA) score is used to assess the morbidity associated with sepsis and incorporates measures of respiratory, hemostatic, liver, cardiovascular, neurological and renal function. Within this study, data collected from experimental sheep over time were collated into a modified SOFA score representing cardiovascular, hemostatic and renal function using human clinical reference ranges in the absence of validated ovine ranges (Table IV). All sheep exhibited an increase in modified SOFA score over time (Fig. 5), however increases were most apparent in sheep administered the highest bacterial dose. These results demonstrate that combined morbidity scores may have value as endpoints for preclinical sepsis studies.

\section{Discussion}

Sepsis still causes significant mortality in developed nations and remains the primary cause of death from infection (22). 

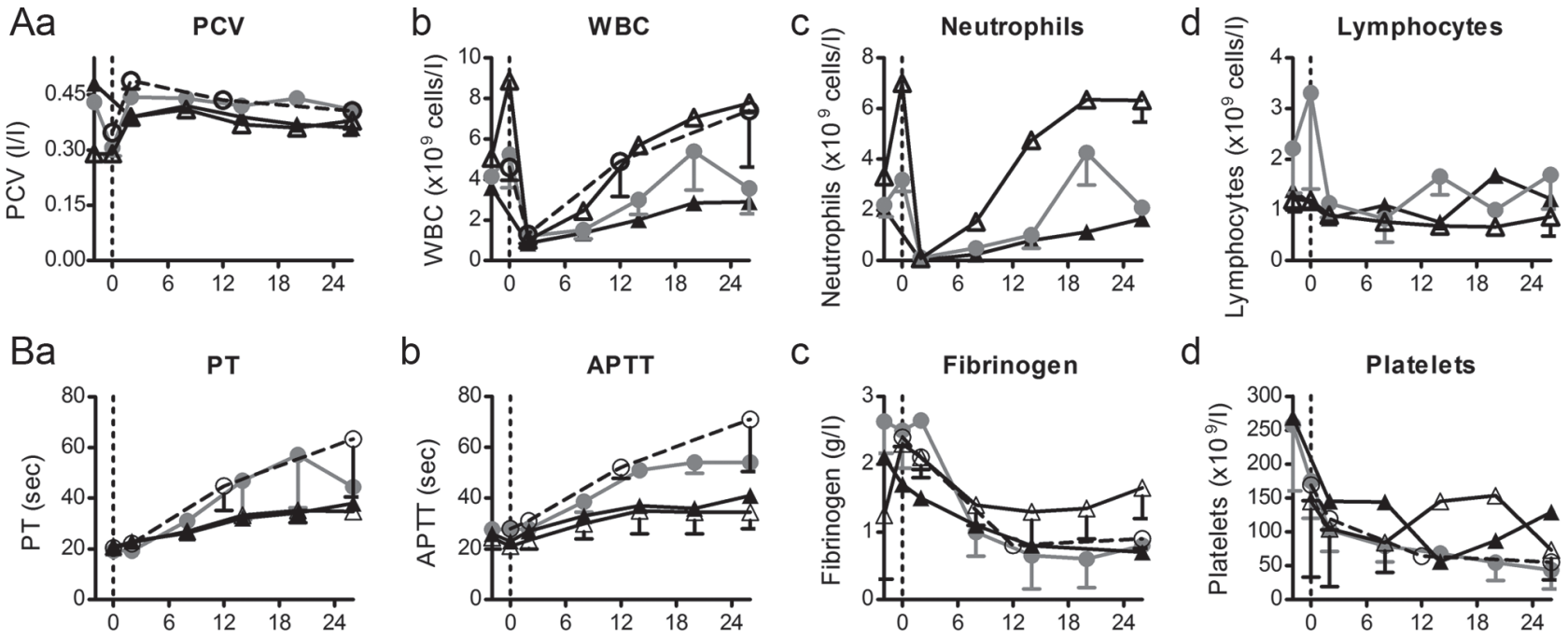

C

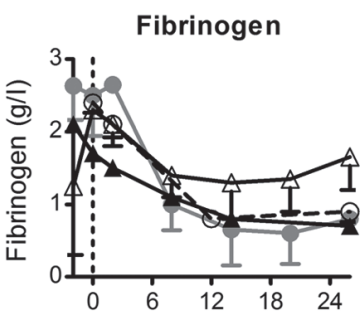

d
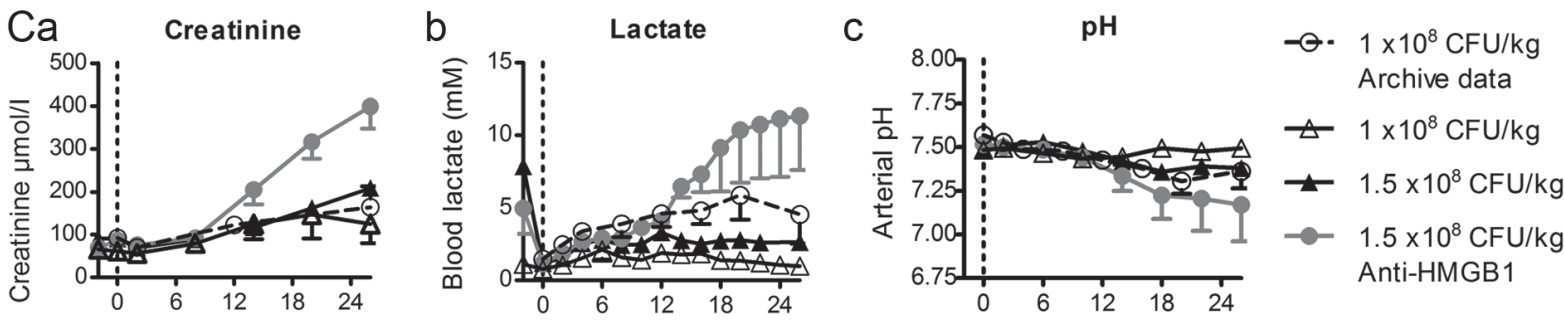

Hours since E Coli

Figure 4. Measured hematological, coagulative and metabolic parameters in experimental sheep. Sheep were administered $1.5 \times 10^{8} \mathrm{CFU} / \mathrm{ml}$ E. coli (IV) to induce sepsis and either treated with anti-HMGB1 $2 \mathrm{~h}$ post challenge $(\mathrm{n}=3)$ or placebo (saline) treated $(\mathrm{n}=1)$. A total of two sheep were administered a lower E. coli dose (1x10 $\left.{ }^{8} \mathrm{CFU} / \mathrm{ml}\right)$. Hematological parameters were measured from whole blood samples collected every $6 \mathrm{~h}$, with (Aa) PCV, (Ab) WBC, (Ac) neutrophil count and (Ad) lymphocyte count are presented. Coagulation tests were performed on plasma samples collected every $6 \mathrm{~h}$, with (Ba) PT, $(\mathrm{Bb})$ APTT, (Bc) fibrinogen level and $(\mathrm{Bd})$ platelet count from whole blood represented. Metabolic parameters were assessed via $(\mathrm{Ca}) \mathrm{creatinine}$ measurement from six-hourly plasma samples with (Cb) lactate measurement from two-hourly snap-frozen plasma samples and $(\mathrm{Cc}) \mathrm{pH}$ assessment via bedside blood gas analysis $(\mathrm{Cc})$. Experimental groups were compared to archive data $(\mathrm{n}=8)$. Data are presented as the mean-standard error of the mean of treatment groups PCV, packed cell volume; WBC, white blood count; PT, prothrombin time; APTT, activated partial thromboplastic time; HMGB1, high mobility group box 1.

The reclassification of sepsis definitions (Sepsis-3) have highlighted the importance of organ dysfunction for diagnosis $^{6}$. Consequently, there is an increased need for animal models than can recapitulate septic organ damage including circulatory, metabolic, renal and coagulative dysfunction and efforts to assess therapeutics should investigate multiple organ systems (8).

Within the present study, the feasibility of using the OSSICS model for evaluating the impact of anti-HMGB1 pAb therapy on physiological manifestations of septic shock was assessed in six sheep. Analysis of banked plasma samples collected from septic and non-septic OSSICS sheep revealed similar septic HMGB1 release profiles to that found in septic humans. Ovine HMGB1 has been demonstrated to have inflammatory effects and release kinetics that mirror those found in humans in studies of chorioamnionitis (23), cell migration (24) and fetal brain injury (25). Consequently, sheep may respond to HMGB1 blockade in the context of sepsis in a similar manner to humans.

Initially, trials aimed to reproduce a sickness severity achieved in previous studies (see references 15 and 16), however administration of the same $E$. coli dose $\left(1 \times 10^{8} \mathrm{CFU} / \mathrm{kg}\right)$ resulted in decreased morbidity with little requirement for NorA. It was subsequently hypothesized that a higher $E$. coli dose $\left(1.5 \times 10^{8} \mathrm{CFU} / \mathrm{kg}\right)$ may increase the sickness severity to a level observed in previous studies, thus the remainder of trials were performed with this higher $E$. coli dose. An increased bacterial dose increased requirement for NorA to maintain blood pressure, considered the primary study endpoint, however these sheep exhibited a very rapid disease course which culminated in two early deaths. Three sheep were treated with anti-HMGB1 antibodies via IV injection at a rate of $10 \mathrm{mg} / \mathrm{kg}$ based on previous murine studies. Anti-HMGB1 therapy was associated with no improvement to MAP, NorA requirement or vascular resistance in anti-HMGB1 pAb treated sheep compared to controls, however a high degree of variation within this group, a low number of subjects and an early death complicated data interpretation. Additional studies incorporating non-specific pAb control groups would provide information as to whether the increased hypotension and morbidity in anti-HMGB1 treated sheep was specific to anti-HMGB1 or a consequence of IV pAb administration in this model.

Kinetic analysis of administered anti-HMGB1 pAb in treated sheep revealed that circulating $\mathrm{pAb}$ concentration decreased over the study period. Similar fluctuations in mAb concentration in the context of tumor therapy have been attributed to several mechanisms including Ab deposition, FcR binding 
Table IV. Parameters used to calculate the modified sequential organ failure assessment for experimental sheep.

\begin{tabular}{|c|c|c|c|c|c|}
\hline \multirow[b]{2}{*}{ Parameter } & \multicolumn{5}{|c|}{ Score assigned } \\
\hline & 0 & 1 & 2 & 3 & 4 \\
\hline Respiratory $\mathrm{PaO}_{2} / \mathrm{FiO}_{2}, \mathrm{mmHg}$ & $>400$ & $\leq 400$ & $\leq 300$ & $\leq 200$ & $\leq 100$ \\
\hline Coagulation platelets, $\times 10^{3} / \mu 1$ & $>150$ & $\leq 150$ & $\leq 100$ & $\leq 50$ & $\leq 20$ \\
\hline $\begin{array}{l}\text { Cardiovascular MAP, mmHg or } \\
\text { NorA, } \mu \mathrm{g} / \mathrm{kg} / \mathrm{min}\end{array}$ & None & MAP $<70$ & & NorA $\leq 0.1$ & Nor $A>0.1$ \\
\hline $\begin{array}{l}\text { Renal Creat, } \mu \mathrm{mol} / \mathrm{l} \text { or urine } \\
\text { output, } \mathrm{ml} / \mathrm{day}\end{array}$ & Creat $<106.1$ & Creat $\geq 106.1$ & Creat $\geq 176.85$ & $\begin{array}{l}\text { Creat } \geq 309.5 \\
\text { or UO }<500\end{array}$ & $\begin{array}{l}\text { Creat } \geq 442.1 \\
\text { or UO }<200\end{array}$ \\
\hline
\end{tabular}

MAP, mean arterial pressure; NorA, noradrenaline; Creat, creatine.

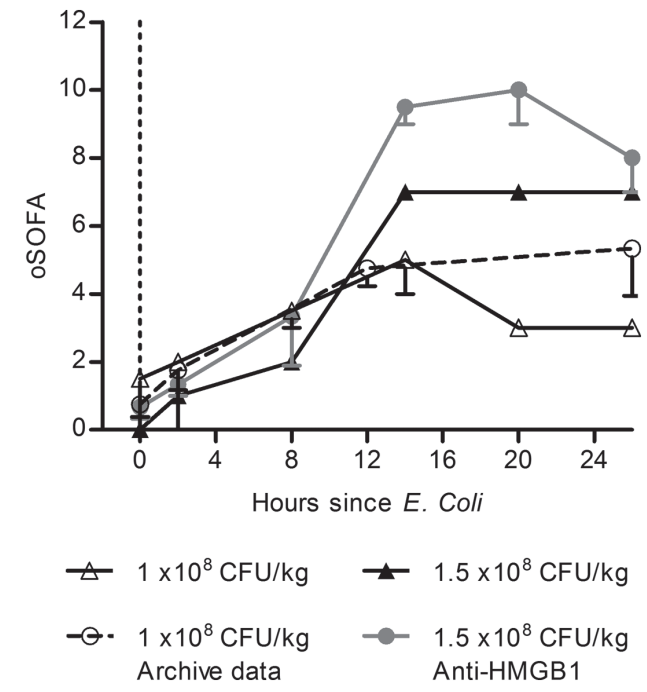

Figure 5. Modified SOFA scores increase over time in the ovine septic shock incorporating intensive care support model. Sheep were administered $1.5 \times 10^{8} \mathrm{CFU} / \mathrm{ml}$ E. coli (IV) to induce sepsis and either treated with anti-HMGB1 two hours post challenge $(n=3)$ or placebo-treated $(n=1)$. A total of two sheep were administered a lower E. coli dose $\left(1 \times 10^{8} \mathrm{CFU} / \mathrm{ml}\right)$. Data representing the degree of cardiovascular hypotension (mean arterial pressure and noradrenaline requirement), coagulation status (platelet count) and renal damage (creatinine) were compiled into a modified SOFA score using human clinical ranges. HMGB1, high mobility group box 1; SOFA, sequential organ failure assessment

or consumption within immune complexes (26). All sheep exhibited vasodilation and developed a positive fluid balance as fluids were administered while urinary production slowed, thus loss of administered pAb into edematous tissues and peritoneal fluid accumulations may have occurred. Homology of HMGB1 between humans and sheep is predicted to be over 98\% (27) thus a commercial ELISA manufactured for measurement of human HMGB1 was able to measure plasma HMGB1 in septic sheep. The administration of anti-HMGB1 pAb did not reduce the detectable plasma HMGB1 in the timeframe however it is not known if detected HMGB1 was bioactive or functionally neutralized as the antibodies used in the ELISA method may bind different epitopes to therapeutic pAbs.

Analysis of hematological parameters within this study supported previous evidence that progressive anemia develops within the OSSICS model (15). Rapid declines in WBC and neutrophil counts occurred upon challenge (28), which failed to recover in groups receiving a high $E$. coli dose. While the magnitude of leukocytosis and neutrophilia have been correlated with the dose of endotoxin in human challenge studies (29), leukopenia has been associated with mortality in gram-negative sepsis in humans (30). Thus, in this model, delayed leukocyte responses may indicate failure to control bacteremia resulting in a more severe septic phenotype. Sheep from previous studies exhibited coagulative deficiency and fibrinogen depletion, however similarly dosed sheep in the current study did not exhibit coagulative deficiency of this degree. This variation between experiments is likely to represent alterations in sheep cohorts, as clotting assays and challenge organism were consistent between the studies. For future studies, additional controls or a rolling control group may be employed to minimize the effect of variation between cohorts. In the current study, anti-HMGB1 pAb treated sheep exhibited the most profound clotting deficiencies, which may be a result of marked fibrinogen depletion (apparent in anti-HMGB1 pAb treated sheep $2 \mathrm{~h}$ following challenge) or the depletion of other clotting factors. Antibody-induced type III hypersensitivity reactions are associated with platelet activation and the formation of clotting-factor depleting microthrombi (31), however intravenous immunoglobulin has also been used to treat sepsis-associated coagulopathy (32). Anti-HMGB1 pAb treated sheep also exhibited increased acidosis, with creatinine and lactate increased compared to other groups. Comparison of the current study to OSSICS sheep administered non-immune ovine pAbs may provide answers as to the potential relationship between coagulopathy, acidosis and organ dysfunction and anti-HMGB1 pAb therapy. The high degree of variation in the OSSICS model compared to murine models does however allow greater comparison to heterogeneous human sepsis, but as with human studies large numbers must be included for reliable conclusions to be drawn (33).

An ideal preclinical sepsis model would incorporate relevant clinical endpoints to enable clinical translation. Clinical endpoints for sepsis RCT's have been under scrutiny (34), and the failure of multiple sepsis-specific therapies to generate meaningful differences in study endpoints highlights the need for the careful endpoint selection. Measures of morbidity during ICU stay are frequently employed as secondary outcomes 
in RCT's (35). In the context of animal models, short-term morbidity endpoints also circumvent ethical and practical issues associated with measuring long-term mortality (2). Clinically, composite morbidity scores such as the SOFA score are used to assess sepsis severity and morbidity (6). Within this study, human ranges for the SOFA score calculation were used to develop a modified SOFA score incorporating measures of cardiovascular, hemostatic and renal dysfunction (Table IV). Modified SOFA scores were calculated for experimental sheep and archive data and septic sheep exhibited increased scores over time, thus a modified SOFA score may provide a useful assessment of sepsis severity. Practical limitations prevent the use of the Glasgow coma scale in the OSSICS model, however future studies could incorporate liver function tests and respiratory measurements for the purpose of developing an ovine SOFA. Inclusion of appropriate reference ranges for physiological criteria and a validated ovine SOFA score equivalent would increase the utility of the model (36). As the highest bacterial dose used in this study caused early mortality in two sheep, a lower bacterial dose or the administration of antibiotics may allow the analysis of morbidity endpoints, an increasingly common outcome in clinical trials (35). Short study periods may only enable evaluation of agents that show marked efficacy in disease, thus a longer septic period may allow the study of more moderate differences between groups. Performing additional tests could provide more insight into the course of OSSICS, such as liver function tests for liver damage and D-dimer assessment for the detection of DIC (37). These foundation studies could help further develop a robust and authentic large animal model for the assessment of immunotherapeutics in sepsis.

The increased focus on organ damage in sepsis monitoring highlights the need for measurement of multiple parameters in preclinical studies ${ }^{8}$. While small animal models are useful for the study of molecular processes and screening potential therapeutics, large animal models remain an important step for the study of physiological processes involving multiple organ systems. While no singular model can possibly recapitulate the complexities encountered in human sepsis, animal models remain a crucial tool for preclinical evaluation of therapeutics. With the refinements, the OSSICS model could become a robust tool in assessing sepsis therapies via multiple organ dysfunction measures and facilitate translation into the clinic by using relevant endpoints. The intelligent and rational optimization of such models may reduce the discrepancy seen in results between preclinical and clinical studies and enable the development of effective host-specific therapeutics targeting sepsis.

\section{Acknowledgements}

The authors would like to thank Dr Susan Porter South (Australian Health and Medical Research Institute, Adelaide, Australia) for her technical expertise and analysis of ovine samples, and Mr. Jason Edwards (Royal Adelaide Hospital, Adelaide, Australia) for his dedicated intensive care unit monitoring.

\section{Funding}

The present study was funded by an Australian Research Council Linkage Project grant (grant no. LP120100606) to
JDH (University of South Australia), MJC (Royal Adelaide Hospital) and TRK (South Australian Health and Medical Research Institute).

\section{Availability of data and materials}

The datasets used and/or analyzed during the current study are available from the corresponding author on reasonable request.

\section{Authors' contributions}

NES acquired and analyzed the data, and wrote the main manuscript text. CHN contributed to the design of the study and data acquisition. CKF contributed to the data analysis and data interpretation, and edited the manuscript. TRK designed the experiments and performed the sheep study. MJM designed the study, interpreted the data and provided archive data. MJC contributed to the design of the study and edited the manuscript. KRD contributed to the design of the study interpreted data, and wrote and edited the main manuscript. JDH contributed to the conception of the study, interpreted the data and edited the manuscript.

\section{Ethical approval and consent to participate}

All sheep studies were approved by The South Australian Health and Medical Research Institute Animal Ethics Committee (Adelaide, Australia) and conducted following institutional and national ethical guidelines according to the 'Australian code for the care and use of animals for scientific purposes (2013)'.

\section{Patient consent for publication}

Not applicable.

\section{Competing interests}

The authors declare that they have no competing interests.

\section{References}

1. Fink MP: Animal models of sepsis. Virulence 5: 143-153, 2014.

2. Nemzek JA, Hugunin KM and Opp MR: Modeling sepsis in the laboratory: Merging sound science with animal well-being. Comp Med 58: 120-128, 2008.

3. Seok J, Warren HS, Cuenca AG, Mindrinos MN, Baker HV, Xu W, Richards DR, McDonald-Smith GP, Gao H, Hennessy L, et al: Genomic responses in mouse models poorly mimic human inflammatory diseases. Proc Natl Acad Sci USA 110: 3507-3512, 2013.

4. Takao K and Miyakawa T: Genomic responses in mouse models greatly mimic human inflammatory diseases. Proc Natl Acad Sci USA 112: 1167-1172, 2014.

5. Di Giantomasso D, Morimatsu H, Bellomo R and May CN: Effect of low-dose vasopressin infusion on vital organ blood flow in the conscious normal and septic sheep. Anaesth Intensive Care 34: 427-433, 2006.

6. Singer M, Deutschman CS, Seymour CW, Shankar-Hari M, Annane D, Bauer M, Bellomo R, Bernard GR, Chiche JD, Coopersmith CM, et al: The third international consensus definitions for sepsis and septic shock (Sepsis-3). JAMA 315: 801-810, 2016.

7. Pickkers $\mathrm{P}$ and Kox M: Towards precision medicine for sepsis patients. Crit Care 21: 11, 2017.

8. Fujishima S: Organ dysfunction as a new standard for defining sepsis. Inflamm Regen 36: 24, 2016. 
9. Foley SR, Solano C, Simonova G, Spanevello MM, Bird RJ, Semple JW, Jackson DE, Schibler A, Fraser JF and Fung YL: A comprehensive study of ovine haemostasis to assess suitability to model human coagulation. Thromb Res 134: 468-473, 2014.

10. Tapia P, Soto D, Bruhn A, Alegría L, Jarufe N, Luengo C, Kattan E, Regueira T, Meissner A, Menchaca R, et al: Impairment of exogenous lactate clearance in experimental hyperdynamic septic shock is not related to total liver hypoperfusion. Crit Care 19: 188, 2015.

11. Murakami K, Bjertnaes LJ, Schmalstieg FC, McGuire R, Cox RA, Hawkins HK, Herndon DN, Traber LD and Traber DL: A novel animal model of sepsis after acute lung injury in sheep. Crit Care Med 30: 2083-2090, 2002.

12. Su F, Wang Z, Cai Y, Rogiers P and Vincent JL: Fluid resuscitation in severe sepsis and septic shock: Albumin, hydroxyethyl starch, gelatin or ringer's lactate-does it really make a difference? Shock 27: 520-526, 2007.

13. Fenhammar J, Rundgren M, Hultenby K, Forestier J, Taavo M Kenne E, Weitzberg E, Eriksson S, Ozenci V, Wernerson A and Frithiof R: Renal effects of treatment with a TLR4 inhibitor in conscious septic sheep. Crit Care 18: 488, 2014.

14. Chapman M, Maiden M, Fraser J, Nash C, Crichton F, Sideris P and Kuchel T: An ovine intensive care model of septic shock. Criti Care 14 (Suppl 1): P4, 2010

15. Maiden MJ, Otto S, Brealey JK, Finnis ME, Chapman MJ, Kuchel TR, Nash CH, Edwards J and Bellomo R: Structure and function of the kidney in septic shock. A prospective controlled experimental study. Am J Respir Crit Care Med 194: 692-700, 2016.

16. Maiden MJ, Chapman MJ, Torpy DJ, Kuchel TR, Clarke IJ, Nash CH, Fraser JD and Ludbrook G: Triiodothyronine administration in a model of septic shock: A randomized blinded placebo-controlled trial. Crit Care Med 44: 1153-1160, 2016.

17. Gentile LF and Moldawer LL: HMGB1 as a therapeutic target for sepsis: It's all in the timing! Expert Opin Ther Targets 18 : 243-245, 2014

18. Andersson U and Tracey KJ: HMGB1 is a therapeutic target for sterile inflammation and infection. Annu Rev Immunol 29: 139-162, 2011.

19. Lakhan N, Stevens NE, Diener KR and Hayball JD: CoVaccine $\mathrm{HT}^{\mathrm{TM}}$ adjuvant is superior to Freund's adjuvants in eliciting antibodies against the endogenous alarmin HMGB1. J Immuno Methods 439: 37-43, 2016.

20. Stevens NE, Chapman MJ, Fraser CK, Kuchel TR, Hayball JD and Diener KR: Therapeutic targeting of HMGB1 during experimental sepsis modulates the inflammatory cytokine profile to one associated with improved clinical outcomes. Sci Rep 7: 5850, 2017.

21. Suda K, Kitagawa Y, Ozawa S, Saikawa Y, Ueda M, Ebina M, Yamada S, Hashimoto S, Fukata S, Abraham E, et al: Anti-high-mobility group box chromosomal protein 1 antibodies improve survival of rats with sepsis. World J Surg 30: 1755-1762, 2006.

22. Fleischmann C, Thomas-Rueddel DO, Hartmann M, Hartog CS, Welte T, Heublein S, Dennler U and Reinhart K: Hospital incidence and mortality rates of sepsis. Dtsch Arztebl Int 113: 159-166, 2016

23. Regan JK, Kannan PS, Kemp MW, Kramer BW, Newnham JP, Jobe AH and Kallapur SG: Damage-associated molecular pattern and fetal membrane vascular injury and collagen disorganization in lipopolysaccharide-induced intra-amniotic inflammation in fetal sheep. Reprod Sci 23: 69-80, 2015.
24. Gabrielyan A, Knaak S, Gelinsky M, Arnhold S and Rösen-Wolff A: Hypoxia-conditioned media allows species-specific attraction of bone marrow stromal cells without need for recombinant proteins. BMC Vet Res 10: 56, 2013.

25. Frasch MG, Szynkaruk M, Prout AP, Nygard K, Cao M, Veldhuizen R, Hammond R and Richardson BS: Decreased neuroinflammation correlates to higher vagus nerve activity fluctuations in near-term ovine fetuses: A case for the afferent cholinergic anti-inflammatory pathway? J Neuroinflammation 13: 103, 2016.

26. Tabrizi M, Bornstein GG and Suria H: Biodistribution mechanisms of therapeutic monoclonal antibodies in health and disease. AAPS J 12: 33-43, 2009.

27. Ito I, Fukazawa J and Yoshida M: Post-translational methylation of high mobility group box 1 (HMGB1) causes its cytoplasmic localization in neutrophils. J Biol Chem 282: 16336-16344, 2007.

28. Jain S, Gautam V and Naseem S: Acute-phase proteins: As diagnostic tool. J Pharm Bioallied Sci 3: 118-127, 2011.

29. Michel O, Nagy AM, Schroeven M, Duchateau J, Nève J, Fondu P and Sergysels R: Dose-response relationship to inhaled endotoxin in normal subjects. Am J Respir Crit Care Med 156: $1157-1164,1997$.

30. Kreger BE, Craven DE and McCabe WR: Gram-negative bacteremia. IV. Re-evaluation of clinical features and treatment in 612 patients. Am J Med 68: 344-355, 1980.

31. Hara T, Shimizu K, Ogawa F, Yanaba K, Iwata Y, Muroi E, Takenaka M, Komura K, Hasegawa M, Fujimoto M and Sato S: Platelets control leukocyte recruitment in a murine model of cutaneous arthus reaction. Am J Pathol 176: 259-269, 2009.

32. Ishikura H, Nakamura Y, Kawano Y, Tanaka J, Mizunuma M, Ohta D, Nishida T and Murai A: Intravenous immunoglobulin improves sepsis-induced coagulopathy: A retrospective, single-center observational study. J Crit Care 30: 579-583, 2015.

33. Fink MP and Warren HS: Strategies to improve drug development for sepsis. Nat Rev Drug Discov 13: 741-758, 2014.

34. Opal SM, Dellinger RP, Vincent JL, Masur H and Angus DC The next generation of sepsis clinical trial designs: What is next after the demise of recombinant human activated protein $\mathrm{C}$ ?* Crit Care Med 42: 1714-1721, 2014

35. Mebazaa A, Laterre PF, Russell JA, Bergmann A, Gattinoni L, Gayat E, Harhay MO, Hartmann O, Hein F, Kjolbye AL, et al: Designing phase 3 sepsis trials: Application of learned experiences from critical care trials in acute heart failure. J Intensive Care 4: 24, 2016.

36. Shrum B, Anantha RV, Xu SX, Donnelly M, Haeryfar SM, McCormick JK and Mele T: A robust scoring system to evaluate sepsis severity in an animal model. BMC Res Notes 7: 233, 2014

37. Sathe PM and Patwa UD: D Dimer in acute care. Int J Crit Illn Inj Sci 4: 229-232, 2014.

This work is licensed under a Creative Commons Attribution-NonCommercial-NoDerivatives 4.0 International (CC BY-NC-ND 4.0) License. 